\title{
Tendências da frequência do consumo de feijão por meio de inquérito telefônico nas capitais brasileiras, 2006 a 2009
}

\author{
Trends in frequency of consumption of beans assessed \\ by means of a telephone survey in Brazilian state capitals \\ between 2006 and 2009
}

Gustavo Velásquez-Meléndez ${ }^{1}$

Larissa Loures Mendes ${ }^{1}$

Milene Cristine Pessoa ${ }^{1}$

Luciana Monteiro Vasconcelos Sardinha ${ }^{2}$

Renata Tiene de Carvalho Yokota ${ }^{3}$

Regina Tomie Ivata Bernal ${ }^{4}$

Deborah Carvalho Malta ${ }^{2}$

\footnotetext{
${ }^{1}$ Escola de Enfermagem, Universidade Federal de Minas Gerais (UFMG). Av. Afredo Balena 190, Centro. 30130-100 Belo Horizonte MG.guveme@ufmg.br ${ }^{2}$ Coordenação Geral de Doenças e Agravos Não Transmissíveis, Secretaria de Vigilância em Saúde, Ministério da Saúde.

${ }^{3}$ Programa de Treinamento em Epidemiologia Aplicada aos Serviços do SUS,

Secretaria de Vigilância em Saúde, Ministério da Saúde, Brasil.

${ }^{4}$ Programa de Pós-

Graduação em Saúde Pública. Universidade de São Paulo (FSP-USP).
}

\begin{abstract}
The scope of this paper was to analyze the trends of frequency of consumption of beans between the years 2006 and 2009 in the Brazilian capitals. This is a historical series using the Vigitel database for all Brazilian state capitals. Consumption of beans was described in terms of relative frequency and the trend was assessed using Poisson regression. Between 65.79\% (2009) and $71.85 \%$ (2006) of participants reported consuming beans five or more days per week. The capitals Goiania, Belo Horizonte, Palmas, Brasilia and Cuiaba were in the highest frequency range of consumption throughout the study period. Individuals with a BMI in the appropriate and low weight category had the highest frequencies of consumption in comparison with the overweight and the obese. A significant reduction trend in the consumption of beans per year of the research, except for the 45 to 54-year-old range, was detected. The conclusion drawn was that there has been a significant reduction in the consumption of beans in the Brazilian population and the adoption of monitoring and incentive policies is necessary due to the benefits of the legume.
\end{abstract}

Key words Consumption of beans, Telephone survey, Trends
Resumo Analisar a tendência da frequência do consumo do feijão nos anos de 2006 a 2009 nas capitais brasileiras. Trata-se de uma série histórica utilizando a base de dados do Vigitel de todas as capitais brasileiras. O consumo de feijão foi descrito por meio da frequência relativa e a tendência foi avaliada utilizando-se regressão de Poisson. O consumo de feijão cinco ou mais vezes por semana variou de $71,85 \%$ (2006) a 65,79 (2009). Na maior faixa de frequência de consumo ao longo de todo o período estudado estão incluídas as capitais Goiânia, Belo Horizonte, Palmas, Cuiabá e Brasília. As pessoas com IMC na categoria adequado/baixo peso apresentaram as maiores frequências de consumo em relação aos indivíduos com sobrepeso e obesidade. Foi observada tendência significativa de redução do consumo regular de feijão segundo ano de realização da pesquisa, exceto para a categoria de idade entre os $45 e$ 54 anos. Ocorreu redução significativa da frequência do consumo do feijão pela população brasileira e a adoção de politicas de monitoramento e incentivo do consumo é necessária em função dos benefícios apresentados pela leguminosa.

Palavras-chave Consumo de feijão, Inquérito telefônico, Tendência 


\section{Introdução}

A população brasileira nas últimas décadas passou por intensas mudanças nos padrões alimentares devido às transformações econômicas, sociais, demográficas e sanitárias como, por exemplo, a intensificação do processo de urbanização, o aumento da renda, a maior participação da mulher no mercado de trabalho e também o aumento do acesso a uma maior variedade de gêneros alimentícios ${ }^{1,2}$.

A dieta da população brasileira, tradicionalmente composta de arroz e feijão, é considerada nutricionalmente adequada e especificamente a leguminosa é uma fonte importante de fibras alimentares, proteínas, folato, zinco e outros nutrientes e o resultado da sua combinação com o cereal é uma refeição de adequado valor nutricional ${ }^{3}$.

Pesquisas nacionais em amostras probabilísticas verificaram que entre 1974 e 2003 esse padrão de consumo passou por mudanças. Nesse período houve redução na aquisição de alimentos típicos da dieta das famílias brasileiras como os alimentos fontes de fibras, e por outro lado, aumento na aquisição de alimentos industrializados com alto teor de gordura saturada e bebidas com elevado teor de carboidratos simples ${ }^{4}$. Os resultados desses estudos foram obtidos por estimativas de dados de disponibilidade de alimentos no domicílio ou de outras técnicas de avaliação de consumo familiar. Entretanto, esses estudos apresentam como potencial limitação a avaliação do consumo por meio de média familiar.

Estudos de consumo que avaliem os indivíduos e as tendências de ingestão de alimentos específicos ou grupos de alimentos são muito escassos no Brasil e estão restritos a populações específicas $^{5-7}$.

Recomendações de uma alimentação saudável para a população brasileira estão consolidadas no Guia Alimentar para a População Brasileira. Este propõe o consumo diário de uma porção de feijão e considera adequados os níveis de consumo médio nacional da leguminosa durante o período de 2002-2003, sendo a meta a manutenção ou aumento do seu consumo ${ }^{8,9}$.

O Ministério da Saúde implantou em 2006 o Sistema Nacional de Vigilância de Doenças Crônicas por Inquérito Telefônico (Vigitel) em todas as 26 capitais de estados brasileiros e no Distrito Federal.

Entre os indicadores monitorados, encontram-se informações sobre alimentação, atividade física, tabagismo, morbidade referida, dentre outros $^{10}$. Assim, o objetivo do presente estudo é analisar a tendência do consumo do feijão nos últimos quatro anos nas capitais brasileiras.

\section{Metodologia}

Trata-se de uma série histórica construída utilizando a base de dados do Vigitel com amostra probabilística da população de adultos de todas as capitais brasileiras, residentes em domicílios servidos por pelo menos uma linha telefônica fixa nos anos de 2006 a $2009^{10}$.

Foram usadas as bases de dados completas do sistema. No ano de 2006 foram entrevistados 54.369 indivíduos; em 2007, 54.251; em 2008, 54.353 e em 2009, 54.367 pessoas responderam às questões do Vigitel.

A frequência do consumo de feijão foi investigada a partir da questão: "Em quantos dias da semana o(a) sr(a) costuma comer feijão?” Em 2006, as opções de resposta foram: "todos os dias (inclusive sábado e domingo)", "5 a 6 dias por semana”, "3 a 4 dias por semana”, "1 a 2 dias por semana" "quase nunca", "nunca", respectivamente. Nos anos de 2007 a 2009, houve inversão nas opções de resposta para: "1 a 2 dias por semana”, "3 a 4 dias por semana", " 5 a 6 dias por semana", "todos os dias (inclusive sábado e domingo)", "quase nunca", "nunca", respectivamente. Com as respostas fornecidas foi criado o indicador de consumo regular de feijão: consumo de feijão em cinco ou mais dias por semana. A taxa de resposta foi de $100 \%$ em todos os anos.

As variáveis sociodemográficas contempladas foram: faixa etária $(18-24,25-34,35-44,45-$ 54, 55-64 e > 65 anos), escolaridade (0-8, 9-11 e > 12 anos de estudo), sexo e cor de pele (branca e não branca). Além disso, foi verificada a frequência de consumo da leguminosa segundo Índice de Massa Corporal - IMC $\left(<25 \mathrm{~kg} / \mathrm{m}^{2}-\right.$ adequado ou baixo peso, $25-29,9 \mathrm{~kg} / \mathrm{m}^{2}$ - Sobrepeso, $>30 \mathrm{~kg} / \mathrm{m}^{2}$ - Obesidade).

O consumo de feijão foi descrito por meio da frequência relativa (\%). O referido consumo nas capitais brasileiras no período estudado foi apresentado por meio de tabela e de mapas segundo faixas de frequência de consumo $(<40,40-60$, $60-80,>90 \%)$ maior ou igual a cinco vezes por semana atribuindo-se cores (escala de cinza) para cada faixa, que foi estendida para toda a área do mapa do estado que a capital representa.

A frequência do indicador foi apresentada também para o total da população estudada segundo variáveis sociodemográficas (idade, sexo, escolaridade, cor de pele) e segundo IMC. As 
análises foram feitas no programa Stata (versão 9.0). Para avaliar mudanças temporais do consumo de feijão no período de 2006-2009 utilizou-se o modelo de regressão de Poisson sendo a variável resposta o consumo regular de feijão em cinco dias da semana ou mais e a variável independente o ano de realização da pesquisa de forma contínua. O nível de significância utilizado foi de $5 \%{ }^{11}$.

As ponderações das estimativas de cada cidade foram compostas pela multiplicação de três fatores: o inverso do número de linhas telefônicas existentes no domicílio do indivíduo entrevistado; o número de pessoas com idade $>18$ anos no domicílio; e o peso pós-estratificação para corrigir a sub ou super-representação de estratos sociodemográficos de acordo com sexo, faixa etária e escolaridade decorrente da cobertura diferencial desses estratos pela rede de telefonia fixa, em relação à população adulta total de cada município, segundo o censo demográfico de 2000. Para as estimativas referentes ao conjunto das 27 cidades, incorporou-se um quarto fator, que considera as diferenças dos contingentes de população e o número semelhante de pessoas entrevistadas em cada cidade ${ }^{10}$.
Por se tratar de entrevista por telefone, o termo de consentimento livre e esclarecido foi substituído pelo consentimento verbal obtido por ocasião dos contatos telefônicos com os entrevistados. Os pesquisadores não tiveram acesso aos nomes dos participantes. O Vigitel foi aprovado pelo Comitê Nacional de Ética em Pesquisa para Seres Humanos do Ministério da Saúde.

\section{Resultados}

No período estudado, entre 65,79\% (2009) e $71,85 \%$ (2006) dos participantes referiram consumir feijão cinco ou mais vezes por semana (Tabela 1).

A Figura 1 mostra o mapa da distribuição do consumo de feijão em todas as capitais brasileiras. Observa-se que na maior faixa de frequência de consumo ao longo de todo o período estudado estão incluídas as capitais Goiânia, Belo Horizonte, Palmas, Cuiabá e Brasília. As capitais com menores frequências de consumo no período de estudo foram Florianópolis, Manaus, Macapá e São Luís. No que diz respeito às regiões, as maiores frequências de consumo foram

Tabela 1. Consumo de feijão em cinco ou mais dias da semana segundo faixa etária, sexo, escolaridade, cor de pele e índice de massa corporal, Brasil, 2006 a 2009.

\begin{tabular}{|c|c|c|c|c|c|}
\hline Variáveis & 2006 & 2007 & 2008 & 2009 & p-valor \\
\hline Total & 71,85 & 67,11 & 66,59 & 65,79 & $<0,001$ \\
\hline \multicolumn{6}{|l|}{ Faixa Etária (anos) } \\
\hline $18-24$ & 75,28 & 66,23 & 69,51 & 65,18 & $<0,001$ \\
\hline $25-34$ & 73,95 & 70,03 & 67,60 & 67,39 & $<0,001$ \\
\hline $35-44$ & 71,98 & 67,98 & 66,06 & 67,32 & $<0,001$ \\
\hline $45-54$ & 68,73 & 65,98 & 65,98 & 66,54 & 0,122 \\
\hline $55-64$ & 69,83 & 65,59 & 65,50 & 63,77 & $<0,001$ \\
\hline 65 e mais & 64,10 & 61,96 & 59,79 & 59,60 & $<0,002$ \\
\hline \multicolumn{6}{|l|}{ Sexo } \\
\hline Masculino & 78,63 & 73,40 & 73,39 & 72,42 & $<0,001$ \\
\hline Feminino & 66,05 & 61,73 & 60,78 & 60,12 & $<0,001$ \\
\hline \multicolumn{6}{|l|}{ Cor de pele } \\
\hline Branco & 66,92 & 63,33 & 62,00 & 61,20 & $<0,001$ \\
\hline Não Branco & 75,10 & 69,51 & 69,29 & 68,43 & $<0,001$ \\
\hline \multicolumn{6}{|l|}{ Escolaridade (anos) } \\
\hline $0-8$ & 77,15 & 71,87 & 71,36 & 70,74 & $<0,001$ \\
\hline $9-11$ & 72,32 & 67,17 & 66,91 & 64,92 & $<0,001$ \\
\hline 12 e mais & 53,94 & 51,72 & 50,71 & 51,45 & 0,02 \\
\hline \multicolumn{6}{|c|}{ Índice de Massa Corporal $\left(\mathrm{kg} / \mathrm{m}^{2}\right)$} \\
\hline$<25$ & 72,85 & 69,00 & 68,21 & 67,68 & $<0,001$ \\
\hline $25-29,9$ & 71,72 & 65,93 & 65,12 & 64,49 & $<0,001$ \\
\hline$>30$ & 66,03 & 63,12 & 62,05 & 62,35 & 0,05 \\
\hline
\end{tabular}



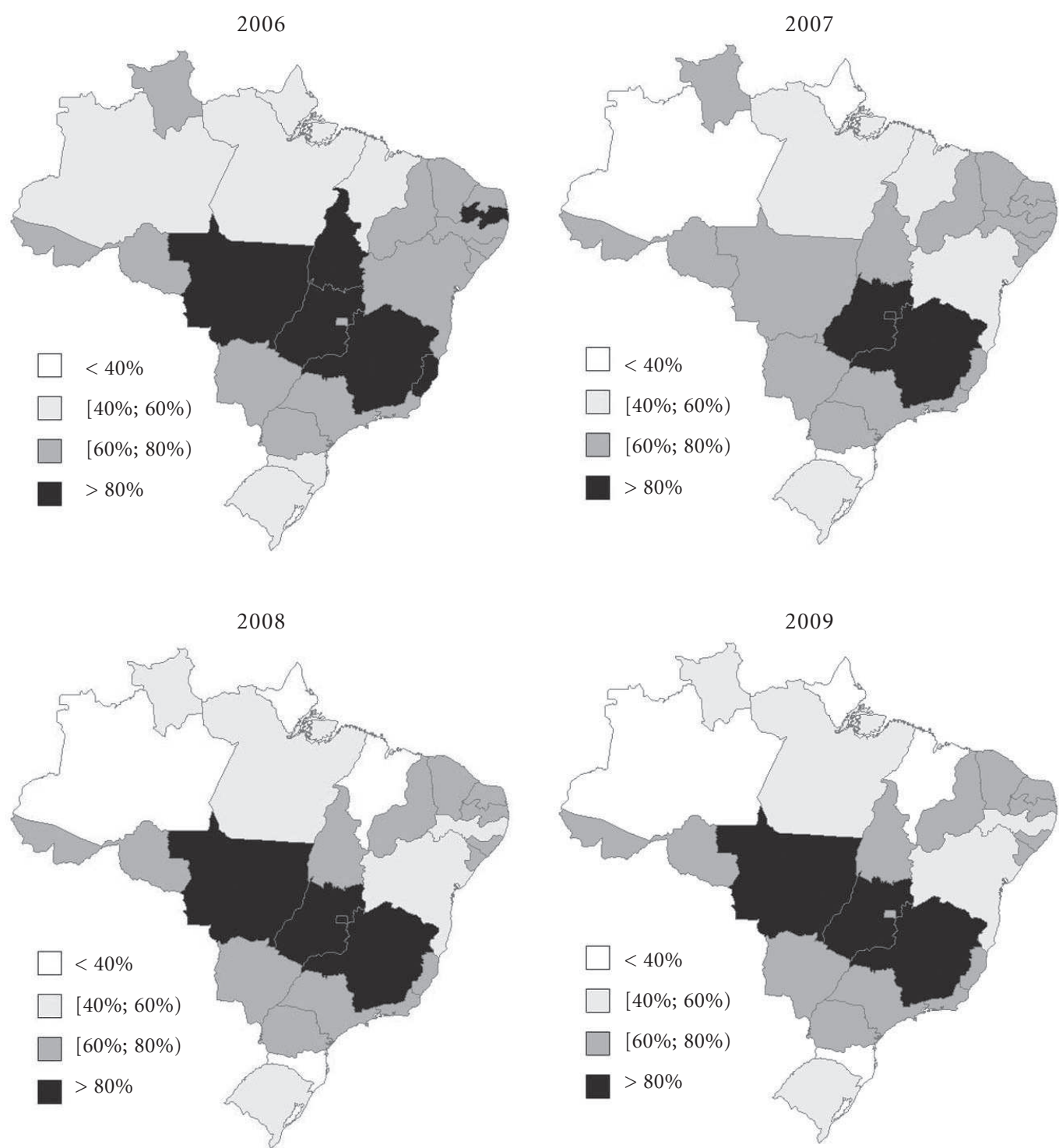

Figura 1. Frequências de consumo de feijão em cinco ou mais vezes por semana estimadas para 2006 a 2009 em adultos com 18 anos ou mais nas capitais, Brasil.

observadas na região Centro-Oeste e as menores na região Sul (Tabela 2).

Foi observada tendência significativa de redução do consumo regular de feijão segundo ano de realização da pesquisa. Essa tendência de diminuição do consumo foi observada em todas as categorias de variáveis consideradas neste estudo, com exceção da faixa de 45 - 54 anos ( $\mathrm{p}=$ $0,122)$ na qual o consumo manteve estabilidade ao longo dos anos estudados (Tabela 1).

A escolaridade foi a variável que melhor mostrou as diferenças de consumo de feijão (cinco ou mais vezes/semana) entre suas respectivas categorias em todos os anos estudados. Os participantes com escolaridade de 12 anos e mais apresentaram as menores porcentagens (aproximadamente 50\%) de consumo regular nos últimos três anos do período estudado. O percentual mais alto de consumo foi observado naqueles com zero a oito anos de estudo, sendo esse padrão similar em todos os anos.

Quanto ao IMC verificou-se que as pessoas com IMC na categoria adequada/baixo peso apresentaram as maiores frequências de consu- 


\begin{tabular}{|c|c|c|c|c|c|c|c|c|}
\hline \multirow[t]{2}{*}{ Regiões/Capitais } & \multicolumn{2}{|r|}{2006} & \multicolumn{3}{|c|}{2007} & \multicolumn{2}{|l|}{2008} & \multirow{2}{*}{$\frac{2009}{\text { IC 95\% }}$} \\
\hline & $\%$ & IC95\% & $\%$ & IC95\% & $\%$ & IC95\% & $\%$ & \\
\hline \multicolumn{9}{|l|}{ Norte } \\
\hline Belém & 55,19 & $52,15-58,20$ & 47,65 & $44,36-50,96$ & 41,63 & $38,23-45,12$ & 40,20 & $36,70-43,80$ \\
\hline Boa Vista & 71,16 & $68,00-74,13$ & 61,84 & $58,12-65,41$ & 52,54 & $48,45-56,60$ & 55,80 & $51,50-60,10$ \\
\hline Macapá & 44,94 & $41,60-48,33$ & 34,94 & $31,41-38,64$ & 35,80 & $32,03-39,76$ & 32,00 & $28,00-36,00$ \\
\hline Manaus & 50,03 & $46,64-53,42$ & 37,82 & $34,68-41,08$ & 38,26 & $34,97-41,67$ & 38,40 & $34,60-42,10$ \\
\hline Palmas & 84,42 & $81,50-86,95$ & 76,52 & $72,84-79,83$ & 73,04 & $68,21-77,38$ & 76,90 & $72,50-81,20$ \\
\hline Porto Velho & 76,23 & $73,42-78,82$ & 71,43 & $68,22-74,44$ & 70,22 & $67,03-73,23$ & 67,00 & $63,50-70,60$ \\
\hline Rio Branco & 74,17 & $70,93-77,15$ & 70,18 & $66,77-73,38$ & 61,93 & $56,22-67,34$ & 65,00 & $60,80-69,20$ \\
\hline \multicolumn{9}{|l|}{ Nordeste } \\
\hline Aracaju & 78,57 & $76,02-80,91$ & 74,81 & $71,70-77,69$ & 72,33 & $68,71-75,67$ & 74,00 & $70,60-77,40$ \\
\hline Fortaleza & 78,64 & $75,99-81,07$ & 71,74 & $68,42-74,84$ & 70,93 & $67,52-74,12$ & 69,10 & $65,60-72,60$ \\
\hline João Pessoa & 80,68 & $78,25-82,90$ & 76,44 & $73,59-79,06$ & 72,42 & $68,66-75,88$ & 73,30 & $69,90-76,70$ \\
\hline Maceió & 75,42 & $72,55-78,08$ & 70,35 & $66,87-73,61$ & 69,08 & $65,16-72,71$ & 70,60 & $66,80-74,50$ \\
\hline Natal & 79,10 & $76,67-81,34$ & 74,52 & $71,32-77,47$ & 71,79 & $68,42-74,93$ & 76,30 & $73,30-79,20$ \\
\hline Recife & 67,83 & $64,98-70,55$ & 66,04 & $62,97-68,99$ & 58,40 & $55,03-61,70$ & 58,50 & $54,80-62,20$ \\
\hline Salvador & 65,85 & $62,84-68,74$ & 58,86 & $55,61-62,03$ & 56,12 & $52,83-59,37$ & 54,70 & $51,20-58,20$ \\
\hline São Luís & 52,61 & $49,44-55,76$ & 41,02 & $37,72-44,40$ & 37,63 & $34,50-40,86$ & 38,40 & $34,90-41,90$ \\
\hline Teresina & 73,61 & $70,47-76,54$ & 66,46 & $63,14-69,63$ & 67,08 & $63,57-70,41$ & 67,30 & $63,80-70,90$ \\
\hline \multicolumn{9}{|l|}{ Centro-Oeste } \\
\hline Brasília & 79,48 & $76,26-82,37$ & 80,97 & $78,55-80,97$ & 80,49 & $78,12-82,65$ & 74,50 & $70,30-78,70$ \\
\hline Campo Grande & 79,26 & $76,70-81,61$ & 76,05 & $72,85-78,98$ & 74,38 & $71,38-77,17$ & 75,40 & $72,50-78,40$ \\
\hline Cuiabá & 84,49 & $82,33-86,43$ & 78,25 & $75,49-80,79$ & 82,43 & $80,16-84,50$ & 82,20 & $79,70-84,70$ \\
\hline Goiânia & 85,78 & $83,68-87,65$ & 81,17 & $78,43-83,64$ & 82,85 & $80,49-84,99$ & 81,70 & $78,20-85,20$ \\
\hline \multicolumn{9}{|l|}{ Sudeste } \\
\hline Belo Horizonte & 85,42 & $83,48-87,16$ & 83,61 & $81,43-85,57$ & 82,18 & $78,83-85,10$ & 82,70 & $80,50-84,90$ \\
\hline Rio de Janeiro & 77,08 & $74,70-79,29$ & 71,32 & $68,21-74,24$ & 73,53 & $71,06-75,86$ & 71,70 & $69,10-74,40$ \\
\hline São Paulo & 72,29 & $69,80-74,65$ & 68,93 & $66,29-71,45$ & 69,32 & $66,50-72,01$ & 68,60 & $65,00-72,20$ \\
\hline Vitória & 83,03 & $81,05-84,85$ & 76,21 & $73,43-78,78$ & 79,96 & $77,59-82,14$ & 76,40 & $73,50-79,40$ \\
\hline \multicolumn{9}{|l|}{ Sul } \\
\hline Curitiba & 64,74 & $62,06-67,33$ & 61,29 & $58,41-64,10$ & 63,95 & $61,07-66,74$ & 65,30 & $62,40-68,20$ \\
\hline Florianópolis & 42,28 & $39,21-45,42$ & 35,31 & $32,26-38,49$ & 36,23 & $33,26-39,31$ & 34,70 & $31,40-38,00$ \\
\hline Porto Alegre & 53,94 & $50,82-57,03$ & 49,05 & $45,98-52,14$ & 49,06 & $45,89-52,24$ & 49,00 & $45,80-52,20$ \\
\hline
\end{tabular}

mo em relação aos indivíduos com sobrepeso e obesidade.

\section{Discussão}

Não foram encontrados na literatura estudos de consumo individual autorreferido em amostras probabilísticas de adultos com foco no consumo de feijão em âmbito nacional. Apenas uma pesquisa de abrangência nacional em população de escolares foi publicada ${ }^{12}$. O feijão é uma leguminosa de participação tradicional na dieta da população brasileira e seu consumo adequado está associado fortemente com a proteção de várias doenças ${ }^{9}$. Os resultados deste estudo ilus- tram e confirmam estimativas prévias de que o consumo do feijão, importante alimento da dieta da população brasileira, ainda é alto, julgado pela frequência de consumo desse alimento em cinco ou mais dias na semana. Esses níveis de consumo variaram de $32,0 \%$ a $85,8 \%$ quando considerada a representação por capitais brasileiras (Tabela 2).

Estudo populacional realizado com mulheres, entre 1995-2005, na cidade de Rio de Janeiro, também mostrou frequências altas do consumo semanal de feijão. Nos períodos de 1995-1996 a frequência foi 9,1 vezes por semana e em 2004-2005 foi de 7,6 vezes por semana. Quando foi avaliado o número de porções os valores foram de 1,8 e 1,3 porções por dia nesses mesmos períodos, respecti- 
vamente, indicando também níveis adequados do consumo de feijão nessa população ${ }^{13}$.

No presente estudo pode-se observar que capitais do Norte e do Sul do país, em geral, apresentam as menores frequências de consumo de feijão, e as capitais do Centro-Oeste e Sudeste as maiores frequências. Como não existem estudos nacionais nos quais o consumo foi aferido em nível individual, as possibilidades de comparação estão limitadas a pesquisas de consumo domiciliar e com populações específicas. Na POF 2002/2003 e na mesma pesquisa realizada em 2008/2009, verificaram-se maiores percentuais de participação de leguminosas no total de calorias determinado pela aquisição alimentar domiciliar na região Nordeste e menores percentuais na região $\mathrm{Sul}^{14,15}$.

Um segundo resultado importante é que o consumo da leguminosa na população brasileira sugere tendência de queda do consumo de feijão ao longo do período estudado (2006-2009). Em pesquisas nacionais anteriores que abrangem períodos mais amplos, mudanças no consumo alimentar da população brasileira foram consolidadas. Ao comparar os dados do Estudo Nacional da Despesa Familiar (ENDEF) de 1974-1975 com pesquisas mais recentes como a Pesquisa de Orçamentos Familiares (POF) 2008/2009, foi observado que nesse período houve um incremento na aquisição de ovos, leite e derivados e substituição de alguns gêneros alimentícios com alto teor de gordura animal por óleos vegetais, margarinas, alimentos preparados e misturas industriais. Detectou-se também uma diminuição no consumo de alimentos tradicionais como arroz, feijão, batata, pão e açúcar ${ }^{14-16}$.

Destaca-se que neste período que o feijão teve sua aquisição, nas áreas urbanas, para consumo no domicílio, reduzido em $49 \%$, passando de 14,7 Kg em 1974/75 para 7,4kg em 2008/2009 ${ }^{15}$.

Ao avaliar a tendência do consumo por categorias de variáveis houve a de queda do consumo para a maioria das faixas etárias, para sexo, cor de pele, escolaridade e para as de IMC adequada/baixo peso e sobrepeso. Porém, a diferença do consumo desta leguminosa deve ser interpretada de forma cautelosa, pois as opções de resposta em 2006, ano com a maior prevalência de consumo regular, foi diferente dos demais, o que pode ter influenciado a comparabilidade do consumo no período estudado.

Observou-se neste estudo que a escolaridade foi a variável cujas categorias mostraram maiores diferenças em relação ao consumo de feijão em todos os anos estudados. Participantes com
12 ou mais anos de escolaridade tiveram as menores frequências de consumo. Esse grupo manteve sua frequência de consumo regular de feijão entre 50,7 e 53,94\% ao longo dos quatro anos de estudo.

A opção por facilidades que poupam tempo de preparo e diminuem a frequência das compras é característica do comensal urbano contemporâneo. Assim, alimentos como o feijão, que demandam tempo de preparo, estão apresentando redução no consumo ${ }^{1}$. Isto é indicativo da mudança no hábito alimentar da população brasileira, uma vez que o consumo de refeições prontas aumentou $80 \%{ }^{14}$ e, por outro lado, houve uma redução na aquisição de gêneros tradicionais como o arroz (com redução do consumo de $60 \%$ ) e o feijão (redução do consumo de $49 \%)^{15}$. Além disso, de acordo com a POF de 2008-2009, houve aumento de $7 \%$ das despesas com alimentação fora do domicílio, quando comparada com POF de 2002-2003 ${ }^{15}$. Provavelmente a população com maior escolaridade, variável proxy de maior renda, represente melhor as mudanças em curso relacionadas tanto a redução do consumo per capita quanto ao percentual das despesas com alimentos tradicionais como arroz e feijão, e consequentemente leve a uma substituição de calorias de alimentos tradicionais por alimentos de rápido preparo, como as massas.

Diferenças no consumo de feijão por escolaridade foram também mostradas na Pesquisa Nacional de Saúde Escolar (PeNSE). Ao comparar escolares de mães com ensino fundamental com escolares de mães com ensino superior, os últimos apresentaram menor chance de consumir feijão em cinco ou mais vezes por semana, e a relação foi oposta quando foi comparado com o consumo de frutas e hortaliças. O consumo frequente de feijão (cinco ou mais vezes por semana) no grupo de escolaridade com nível superior foi de $68 \%$ contra $72,5 \%$ no grupo de escolaridade mais baixa. $\mathrm{O}$ consumo de feijão na população total de escolares foi de $62,6 \%{ }^{12}$.

Estudos provenientes de outras populações como a americana mostram que o consumo de leguminosas é baixo; pesquisas com amostras probabilísticas estimam que o consumo diário referido desse grupo de alimentos atingiu 14\% em 19941996, diminuindo para 7,9\% em 1999-2002. Em 1999-2002 adultos americanos consumiam 0,1 a 0,3 porções por dia, muito abaixo das recomendações para o consumo desse alimento ${ }^{17}$.

Dois aspectos interessantes podem ser destacados a partir dos resultados: o primeiro referese ao fato de que o feijão, provavelmente um dos 
alimentos da dieta da população brasileira com quantidade proporcionalmente maior de fibras alimentares, quando comparado a outros alimentos, constitui um alimento importante no consumo alimentar saudável ${ }^{14}$. Existem evidências de que o consumo de frutas, hortaliças, cereais integrais e leguminosas está associado a efeitos protetores para doenças cardiovasculares e certos tipos de câncer ${ }^{18}$.

Além disso, alguns estudos nacionais mostraram os efeitos benéficos do consumo de feijão em nível populacional. Por exemplo, de acordo com análises de dados de amostra probabilística da população do nordeste e sudeste do Brasil, o consumo de dieta com arroz e feijão confere efeito protetor contra o ganho de peso ${ }^{19}$. Em outro estudo transversal com amostra probabilística realizado com a população americana, ao comparar o grupo que não consumia feijão com os que consumiam, estes últimos apresentaram elevado consumo de fibra alimentar, potássio, magnésio, ferro e cobre, nutrientes importantes na regulação da pressão arterial ${ }^{20}$. Adicionalmente, os consumidores de feijão tiveram uma redução de $23 \%$ do risco de apresentar circunferência da cintura aumentada e além de apresentarem menor peso ${ }^{21}$. Também foi mostrada associação inversa em estudo longitudinal do consumo de leguminosas com câncer de intestino ${ }^{22}$. Contrariamente a esses achados, o padrão tradicional de consumo alimentar da população de Porto Rico, composto basicamente de leguminosas e cereais, foi associado a maior frequência de síndrome metabólica ${ }^{23}$.

Podem ser mencionadas algumas limitações do presente estudo, aquelas que são inerentes a sua proposta metodológica, principalmente decorrente da aferição autorreferida do consumo de alimentos por telefone (viés de informação, viés de memória), e também a ausência de dados relativos à frequência de consumo diário de feijão e ao tamanho da porção consumida. Mencionam-se ainda outras limitações relativas à amostra, composta de residentes em domicílios com linha telefônica fixa, sendo sua representatividade ajustada na análise com a composição sociodemográfica da população brasileira. Além disso, outra limitação metodológica foi o tempo de estudo (2006 a 2009), em que há disponibilidade de informações.

Conclui-se que durante o período estudado a frequência do consumo de feijão na população das capitais brasileiras, apesar da tendência de queda, entre 2006 e 2009, ainda é relativamente alta. Essa tendência aparece consolidada desde anos anteriores no quais vem observando-se redução na aquisição desse alimento revelada pela POF 2008/2009. Cabe destacar que o sistema Vigitel refere-se a populações de adultos e das capitais brasileiras. Mudanças realizadas nas opções de respostas da pergunta sobre o consumo desse alimento na maioria dos anos estudados podem ter introduzido vieses nas respostas, portanto, exigem cautela na extrapolação dos resultados.

O monitoramento do seu consumo torna-se um instrumento valioso em função dos benefícios desta leguminosa. Além disso, a adoção de medidas de incentivo ao consumo de feijão deve ser estimulada, uma vez que tal alimento possui efeitos benéficos pelos nutrientes que o compõe como fibras alimentares, ácido fólico, zinco e magnésio, e o seu consumo está associado a baixo risco para várias doenças crônicas. Recomenda-se a realização de estudos adicionais para determinar se seu consumo associa-se a padrões de consumo de outros grupos de alimentos.

\section{Colaboradores}

G Velásquez-Meléndez foi responsável pela concepção e projeto, interpretação dos dados, redação do artigo e revisão crítica relevante do conteúdo intelectual, aprovação da versão a ser publicada. LL Mendes realizou análise e interpretação dos dados, redação do artigo e revisão crítica relevante do conteúdo intelectual. MC Pessoa realizou análise e interpretação dos dados, revisão crítica relevante do conteúdo intelectual. LMV Sardinha e RTC Yokota fizeram revisão crítica relevante do conteúdo intelectual. RTI Bernal realizou análise e interpretação dos dados e DC Malta foi responsável pela concepção e projeto, revisão crítica relevante do conteúdo intelectual e aprovação da versão a ser publicada.

\section{Agradecimentos}

À Secretaria de Vigilância em Saúde, Ministério da Saúde (SVS/MS) pelo financiamento do projeto. 
Referências

1. Schlindwein MM, Kassouf AL. Análise da influência de alguns fatores socioeconômicos e demográficos no consumo domiciliar de carnes no Brasil. Rev Econ Sociol Rural 2006; 44(3):549-572.

2. Peña M, Bacallao J. La obesidade en la pobreza: un problema emergente en las Américas. In: Organización Panamericana de la Salud (OPAS). La obesidad en la pobreza: un nuevo reto para la Salud $\mathrm{Pu}$ blica. Washington, DC: OPAS; 2000. p. 3-11.

3. Messina MJ. Legumes and soybeans: overview of their nutritional profiles and health effects. Am J Clin Nutr 1999; 70(Supl.3):S439-S450.

4. Levy-Costa RB, Sichieri R, Pontes NS, Monteiro CA. Disponibilidade domiciliar de alimentos no Brasil: distribuição e evolução (1974-2003). Rev Saude publica 2005; 39(4):530-540.

5. Mattos LL, Martins IS. Consumo de fibras alimentares em população adulta. Rev Saude publica 2000; 34(1):50-55.

6. Neumann AICP, Martins IS, Marcopito LF, Araujo EAC. Padrões alimentares associados a fatores de risco para doenças cardiovasculares entre residentes de um município brasileiro. Rev Panam Salud Publica 2007; 22(5):329-339.

7. De Bem Lignani J, Sichieri R, Burlandy L, SalesCosta R. Changes in food consumption among the Programa Bolsa Família participant families in Brazil. Public Health Nutr 2010; 14(5):785-792.

8. Brasil. Ministério da Saúde (MS). CoordenaçãoGeral da Política de Alimentação e Nutrição. Secretaria de Atenção à Saúde. Guia alimentar para a população brasileira: promovendo a alimentação saudável. Brasília: MS; 2006.

9. Sichieri R, Coitinho DC, Monteiro JB, Coutinho WF. Recomendações de alimentação e nutrição saudável para a população brasileira. Arq Bras Endocrinol Metab 2000; 44(3):227-232.

10. Brasil. Ministério da Saúde (MS). Secretaria de Vigilância em Saúde. Secretaria de Gestão Estratégica e Participativa. VIGITEL Brasil 2009: Vigilância de fatores de risco e proteção para doenças crônicas por inquérito telefônico: Estimativas sobre frequência e distribuição sócio-demográfica de fatores de risco e proteção para doenças crônicas nas capitais dos 26 Estados brasileiros e no Distrito Federal em 2009. Brasília: MS, Secretaria de Vigilância em Saúde, Secretaria de Gestão Estratégica e Participativa; 2010. (Série G. Estatística e Informação em Saúde).

11. Moura EC, Morais Neto OL, Malta DC, Moura L, Silva NN, Bernal R, Claro RM, Monteiro CA. Vigilância de Fatores de Risco para Doenças Crônicas por Inquérito Telefônico nas capitais dos 26 estados brasileiros e no Distrito Federal (2006). Rev Bras Epidemiol 2008; 11(Supl. 1):20-37.

12. Levy RB, Castro IRR, Cardoso LO, Tavares LF, Sardinha LMV, Gomes FS, Costa AWN. Consumo e comportamento alimentar entre adolescentes brasileiros: Pesquisa Nacional de Saúde do Escolar (PeNSE), 2009. Cienc Saude Colet 2010; 15(Supl. 2):3085-3097
13. Pereira RA, Andrade RG, Sichieri R. Mudanças no consumo alimentar de mulheres do Município do Rio de Janeiro, Brasil, 1995-2005. Cad Saude publica 2009; 25(11):2419-2432.

14. Instituto Brasileiro de Geografia e Estatística (IBGE). Pesquisa de orçamentos familiares 2002-2003: análise da disponibilidade domiciliar de alimentos e do estado nutricional no Brasil. Rio de Janeiro: IBGE; 2004.

15. Instituto Brasileiro de Geografia e Estatística (IBGE). Pesquisa de orçamentos familiares 2008-2009: análise da disponibilidade domiciliar de alimentos e do estado nutricional no Brasil. Rio de Janeiro: IBGE 2010.

16. Monteiro CA, Mondini L, Levy-Costa RB. Mudanças na composição e adequação nutricional da di eta familiar nas áreas metropolitanas do Brasil (19881996). Rev Saude publica 2000; 34(3):251-258.

17. Mitchell DC, Lawrence FR, Hartman TJ, Curran JM. Consumption of dry beans, peas, and lentils could improve diet quality in the US population. $J$ Am Diet Assoc 2009; 109(5):909-913.

18. Skerrett PJ, Willett WC. Essentials of healthy eating: a guide. J Midwifery Womens Health 2010; 55(6):492501

19. Sichieri R, Castro JFG, Moura AS. Fatores associados ao padrão de consumo alimentar da população brasileira urbana. Cad Saude Publica 2003; 19(Supl. 1):47-53.

20. He FJ, Markandu ND, Coltart R, Barron J, MacGregor GA. Effect ofshort-term supplementation of potassium chloride and potassiumcitrate on blood pressure in hypertensives. Hypertension 2005; 45(4):571-574.

21. Papanikolaou Y, Fulgoni VL. Bean consumption is associated with greater nutrient intake, reduced systolic blood pressure, lower body weight, and a smaller waist circumference in adults: results from the National Health and Nutrition Examination Survey 1999-2002. J Am Coll Nutr 2008; 27(5):569-576.

22. Schatzkin A, Park Y, Leitzmann MF, Hollenbeck AR, Cross AJ. Prospective study of dietary fiber, whole grain foods, and small intestinal cancer. Gastroenterology 2008; 135(4):1163-1167.

23. Noel SE, Newby PK, Ordovas JM, Tucker KL. A traditional rice and beans pattern is associated with metabolic syndrome in Puerto Rican older adults. $J$ Nutr 2009; 139(7):1360-1367.

Artigo apresentado em 05/08/2011

Aprovado em 01/11/2011

Versão final apresentada em 13/11/2011 